\title{
Equivalences (i-MLD) of Tilings and Tiling Spaces
}

\section{Alzahrani $\mathbf{H}^{*}$}

Departmemt of Mathematics, Albaha University, Prince Mohammad Bin Saud, Al Bahah 65527, Saudi Arabia

\begin{abstract}
What does it mean when we say that two simple tilings or two simple tiling spaces are equivalent? There are several different notions that explain this, as we will see in this article.

By introducing a topology on tiling spaces, there is a notion of a continuous map $f: \Omega \rightarrow \Omega$ between two tiling spaces $\Omega, \Omega^{\prime}$. The map $f$ is a homeomorphism if $f$ is $1-1$, onto and $f^{-1}$ is also continuous.

For a homeomorphism between simple tiling hulls, we only need to check whether $f$ is continuous, $1-1$ and onto, since $f^{-1}$ is automatically continuous as $\Omega_{\mathrm{T}}$ is compact.

Next, we want to consider continuous maps respectively homeomorphisms, which interact properly with the action of the isometry group on the tiling spaces.
\end{abstract}

Keywords: Homeomorphism; Conjugacy; Translationally; Euclidean

\section{Introduction}

\section{Topological isometric conjugacy}

Originally, such factor maps were defined only using translations [1]. When requiring $f(\phi(T))=\phi(f(T))$ for arbitrary isometries, many homeomorphisms, which are a topological conjugacy for translations, are no longer topological isometric-conjugacies. The reason is that general isometries do not commute with each other, but translations do $[2,3]$.

Example 1.1: Consider the map $f$ of a tiling space $\Omega$ onto itself, such that $f(\phi(T))=\phi(T)$ for $\phi \in I \operatorname{som}\left(\mathbb{E}^{\mathrm{n}}\right)$, and $\mathrm{T} \in \Omega[4]$ :

For example, if $\mathrm{T}$ is the standard lattice in $\mathbb{E}^{2}$ and $\Omega_{\mathrm{T}}$ its hull (with respect to isometries). Choose $\phi$ as a translation by $v$, that is, $f: T^{\prime} \rightarrow T^{\prime}+v$ for any $T^{\prime} \in \Omega_{\mathrm{T}}[5]$. It is clear that $f$ is a factor map with respect to translations, since $f\left(T^{\prime}+w\right)=T^{\prime}+w+v=f\left(T^{\prime}\right)+w$. On the other hand, $f(\psi(T)) \psi(T)+v) \neq \psi(T+v)$, if $\psi$ is a rotation by $90^{\circ}$ around $(0,0)$, and $v=\left(\frac{1}{4}, \frac{1}{4}\right)$ : Then $(T)=T$ implies that $\psi(T)+v=T+v$, but $\psi(T+v) \neq T+v$,

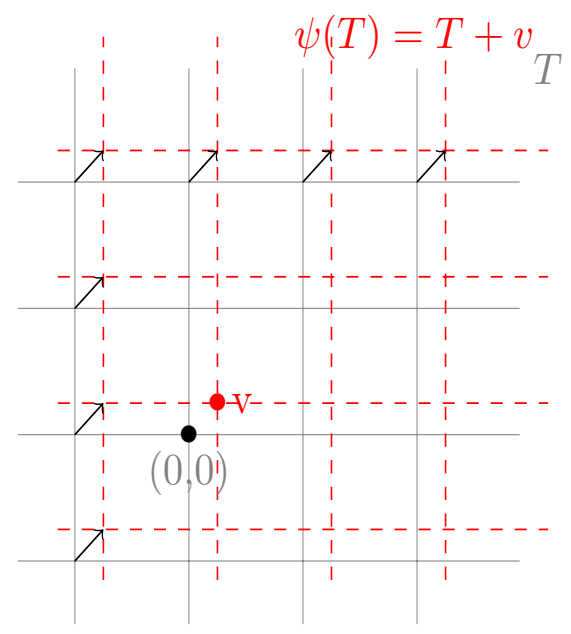

Figure 1: Translated standard lattice tilings of $\mathbb{E}^{2}$. which means that $f$ is not a factor map with respect to isometries (Figure 1).

Remark 1.2 : If we allow for an additional isometry on the affine space $\mathbb{E}^{\mathrm{n}}$ (in our case, just $\phi$ ), then $\Omega_{\mathrm{T}}[6]$ and $\Omega_{\mathrm{T}}$, become topologically conjugated again (proposition 2.7).

This motivates the following definition [7]:

Definition 1.3: For tiling spaces $\Omega$ and $\Omega^{\prime}$, the continuous map $f: \Omega \rightarrow \Omega^{\prime}$ is called an isometric-factor map ( $\gamma$-i-factor map for short) if there exists $\gamma \in \operatorname{Isom}\left(\mathbb{E}^{\mathrm{n}}\right)$, such that for all $\phi \in \operatorname{Isom}\left(\mathbb{E}^{\mathrm{n}}\right)$, and for all $\mathrm{T} \in \Omega$ [8]:

\section{$f(\phi(T))=\left(\gamma \phi \gamma^{-1}\right)(T)$}

If $f$ is also a homeomorphism, then $f$ is called a topological isometricconjugacy (topological $\gamma$-i-conjugacy for short) [9].

Lemma 1.4: The inverse map $f^{-1}: \Omega^{\prime} \rightarrow \Omega$ of a topological $i$-conjugacy, $f: \Omega \rightarrow \Omega^{\prime}$ is also a topological $i$-conjugacy [10].

Proof: For $T^{\prime} \in \Omega^{\prime}$, there exists a unique $T \in \Omega$, such that $f(T)=T^{\prime}$ (as $f$ is a homeomorphism). $f$ is a topological i-conjugacy; therefore, there exists $\gamma \in \operatorname{Isom}\left(\mathbb{E}^{\mathrm{n}}\right)$ such that [11]

$$
\begin{aligned}
f\left(\phi^{\prime}\left(T^{\prime}\right)\right) & =\left(\gamma^{-1} \phi^{\prime} \gamma\right)\left(T^{\prime}\right) ; \forall \phi^{\prime} \in \operatorname{Isom}\left(\mathbb{E}^{n}\right) \\
& \Leftrightarrow \phi^{\prime}\left(T^{\prime}\right)=f^{-1}\left(\left(\gamma^{-1} \phi^{\prime} \gamma\right)\left(T^{\prime}\right)\right) \\
& \Leftrightarrow \gamma \phi \gamma^{-1}\left(T^{\prime}\right)=f^{-1}\left(\phi\left(T^{\prime}\right)\right) ; \operatorname{as} \phi^{\prime}=\gamma \phi \gamma^{-1} .
\end{aligned}
$$

By choosing $\gamma^{\prime}=\gamma^{-1}$, we get:

$$
f^{-1}\left(\phi\left(T^{\prime}\right)\right)=\left(\gamma^{\prime-1} \phi \gamma^{\prime}\left(T^{\prime}\right)\right) ; \forall T^{\prime} \in \Omega^{\prime} .
$$

Hence, $f^{1}$ is a topological $\gamma^{-1}-i$-conjugacy.

*Corresponding author: Alzahrani $\mathrm{H}$, Departmemt of Mathematics, Albaha University, Prince Mohammad Bin Saud, Al Bahah 65527, Saudi Arabia, Tel: +966555007754; E-mail: hawazin03@hotmail.com

Received January 01, 2019; Accepted January 11, 2019; Published January 19 2019

Citation: Alzahrani H (2019) Equivalences (i-MLD) of Tilings and Tiling Spaces. J Appl Computat Math 8: 434. doi: 10.4172/2168-9679.1000434

Copyright: (c) 2019 Alzahrani H. This is an open-access article distributed under the terms of the Creative Commons Attribution License, which permits unrestricted use, distribution, and reproduction in any medium, provided the original author and source are credited. 
Remark 1.5 If we define topological conjugacies using only translations, the definition reduces to [12]:

\section{$\forall \tau \in \operatorname{Trans}\left(\mathbb{E}^{\mathrm{n}}\right), f(T+\tau)=f(T)+\tau$}

Since conjugating in the abelian group, $\operatorname{Trans}\left(\mathbb{E}^{\mathrm{n}}\right)$ is trivial. One could also allow for automorphisms of Isom $\left(\mathbb{E}^{\mathrm{n}}\right)$ different from $\varphi \rightarrow \gamma^{-1} \varphi \gamma$ (if they exists at all). These notions of topological conjugacy on tiling spaces are different, as example 1.1 shows.

Example 1.6: Let $T$ be the standard lattice tiling constructed from tiles which are squares with vertices of the form $(n+m),(n+1, m)$, $(\mathrm{n}, \mathrm{m}+1),(\mathrm{n}+1, \mathrm{~m}+1) ; \mathrm{n}, \mathrm{m} \in \mathbb{Z}$ and $\mathrm{T}^{\prime}$ the slanted lattice tiling obtained from the rhomb with vertices $(0,0),(1,0),(2,1),(1,1)$ and all its translations by vectors $(n, m) \in \mathbb{Z}^{2}$, in Figure 2 .

There is a natural map $f: \Omega_{\mathrm{T}^{\prime}} \rightarrow \Omega_{T}$, such that $f$ is a 4-1 map.

Given a tiling $\bar{T}^{\prime} \in \Omega_{T^{\prime}}$, we obtain a tiling $\bar{T} \in \Omega_{T}$ by halving each of the rhombs in $\bar{T}^{\prime}$ and uniting two of these halves to a square whenever they meet face-to-face along a diagonal edge.

The following picture shows how we obtain the same standard lattice tiling from 4 different slanted lattice tilings:

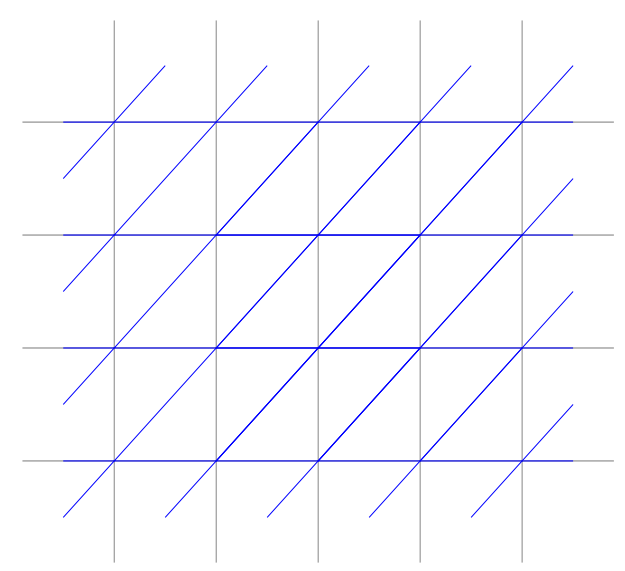

Figure 2: Standard lattice tiling and slanted lattice tiling of $\mathbb{E}^{2}$

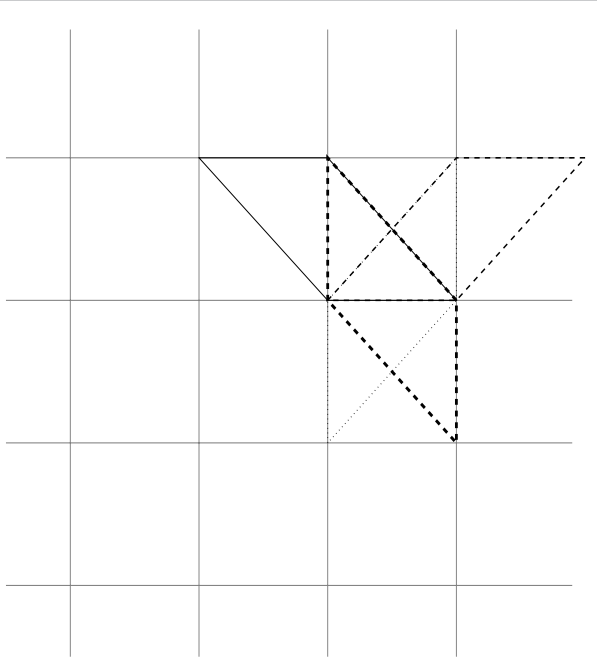

Figure 3: Standard lattice tile can be obtained from one of these four different slanted lattice tiles.
The four slanted lattice tiles in Figure 3, are mapped to each other by isometries in the cosets of $D_{2}$ in $D_{4}$.

Obviously, halving the rhombs commutes with applying an isometry on them, so that $f$ is an i-factor map (with $\gamma=i d \mathbb{E}^{2}$ ).

\section{Mutual Local Derivability}

The strongest notion of equivalence used in the literature is Mutual Local Derivability; (MLD for short).

To obtain our results; we have to extend the definition of MLD [1] to isometrical MLD (Isometrical case) by imposing the taxicab metric $d_{0}$ on $\operatorname{Isom}\left(\mathbb{E}^{\mathrm{n}}\right)$, then replacing translations by isometries in the original definition, as we will see.

\section{MLD tiling spaces}

Definition 2.1: If $\Omega$ and $\Omega^{\prime}$ are tiling spaces, we say that $\Omega^{\prime}$ is isometric locally derivable ( $\gamma-i-\mathrm{LD}$ for short) from $\Omega$ if there is a surjective $\gamma$-i-factor map $f: \Omega \rightarrow \Omega^{\prime}$ that is defined locally. More precisely, there exists a radius $R$ such that, whenever two tilings $T_{1}, T_{2} \in \Omega$ agree on a ball of radius $R$ around $x \in \mathbb{E}^{\mathrm{n}}$, the tilings $f\left(T_{1}\right)$ and $f\left(T_{2}\right)$ in $\Omega^{\prime}$ agree on the patches covering $\gamma(x)$, i.e.,

$$
\left[T_{1}\right]_{B_{R}(x)}=\left[T_{2}\right]_{B_{R}(x)} \text { then }\left[f\left(T_{1}\right)\right]_{\{\gamma(x)\}}=\left[f\left(T_{2}\right)\right]_{\{\gamma(x)\}} .
$$

If these implications hold, $R$ will be called the $i-L D$ radius.

If $f^{-1}$ is a topological $\gamma^{-1} i$-conjugacy making $\Omega \gamma^{-1}$ - i-LD from $\Omega^{\prime}$, then $\Omega$ and $\Omega^{\prime}$ are called $i-M L D$.

Remark 2.2: To distinguish between translationally and isometrically MLD, we add translationally and isometrically.

If we only speak of MLD, we always mean isometrically-MLD.

Similarly, for $\gamma$-LD, we often leave out $\gamma$ if it is clear from the context what $\gamma$ is.

Example 2.3: Consider the standard lattice tiling $T$ in the Euclidean plane. By dividing the tiling $T$ into halves, we get a new tiling $T^{\prime}$ (Figure 4). This gives a natural map $f$ between the tiling spaces $\Omega_{\mathrm{T}}$ and $\Omega_{\mathrm{T}^{\prime}}$ by setting $f(\phi(T))=\phi\left(\mathrm{T}^{\prime}\right)$ for all $\phi \in \operatorname{Isom}\left(\mathbb{E}^{\mathrm{n}}\right)$, since the hull $\Omega_{\mathrm{T}}$ of $T$ and $\Omega_{\mathrm{T}}$ of $T^{\prime}$ are equal to the orbits of $T$ and $T^{\prime}$.

Clearly, $f$ is continuous and an i-factor map (using $\gamma=\mathrm{id}_{2}$ ), as its defining equations shows.

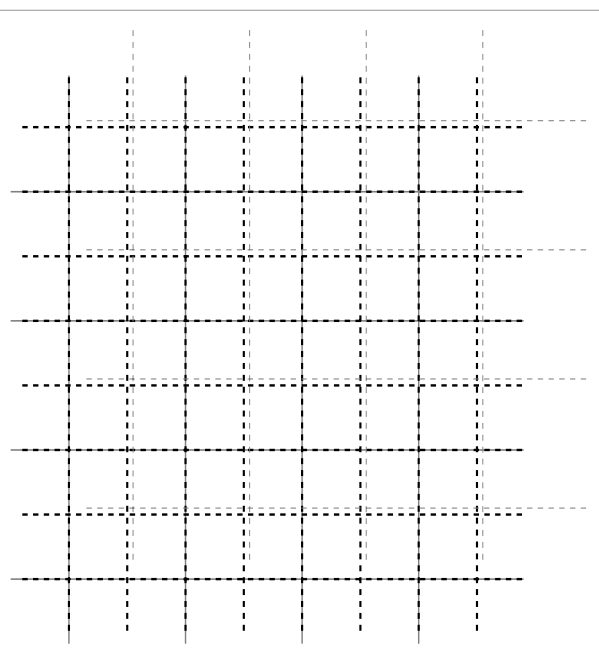

Figure 4: LD but not MLD lattice tilings $\left(T=-, T^{\prime}=---, \bar{T}=---\right)$. 
Also, $f$ is surjective, but is not a homeomorphism as it is not $1-1$, since a given tiling in $\Omega_{\mathrm{T}^{\prime}}$ can be obtained from two tilings $T, \bar{T}$ in $\Omega_{\mathrm{T}}$ differing by a translation of length $\frac{1}{2}$ (Figure 4 ). Therefore, $f(\phi(T))=f((T))$ and $\phi \neq \psi$. The $i$-LD property is satisfied because we can obtain the red tile by taking one of the black tiles and subdividing it, which is a local condition. Then, we can find a radius $R$ around $x \in \mathbb{E}^{\text {n }}$, such that:

$$
[T]_{B_{R}(x)}=\left[T^{\prime}\right]_{B_{R}(x)}[f(T)]_{\{x\}}=\left[f\left(T^{\prime}\right)\right]_{\{x\}} .
$$

For example, $\mathrm{R}>2$ will do, because then, $\mathrm{B}_{\mathrm{R}}(x)$ will always contain a square of any tiling in $\Omega_{\mathrm{T}}$, for any $x \in \mathbb{E}^{2}$.

Lemma 2.4: If $\Omega$ and $\Omega^{\prime}$ are two $i-M L D$ tiling spaces, with $i-M L D$ radius $R$, then, for tilings $T_{1}, T_{2} \in \Omega$,

$$
\left[T_{1}\right]_{B_{r+R}(x)}=\left[T_{2}\right]_{B_{r+R}(x)}\left[f\left(T_{1}\right)\right]_{B_{r}(\gamma(x))}=\left[f\left(T_{2}\right)\right]_{B_{r}(\gamma(x))} \text {; for all } \mathrm{r} \geq 0 .
$$

Proof: By covering the ball $\mathrm{B}_{\mathrm{r}+\mathrm{R}}(x)$ with balls $B_{R}\left(x^{\prime}\right)$ where $\mid x^{\prime}$ $x \mid<\mathrm{r} \Leftrightarrow \mathrm{x}^{\prime} \in \mathrm{B}_{\mathrm{r}}(x)$, we will have:

$$
\begin{aligned}
{\left[T_{1}\right]_{B_{r+R}(x)}=\left[T_{2}\right]_{B_{r+R}(x)} } & \Rightarrow\left[T_{1}\right]_{B_{R}\left(x^{\prime}\right)}=\left[T_{2}\right]_{B_{R}\left(x^{\prime}\right)} ; \quad \forall x^{\prime} \in B_{r}(x) \\
& \Rightarrow\left[f\left(T_{1}\right)\right]_{\left\{x^{\prime}\right\}}=\left[f\left(T_{2}\right)\right]_{\left\{x^{\prime}\right\}} ; \forall x^{\prime} \in B_{r}(\gamma(x)) \\
& \Rightarrow \bigcup_{x^{\prime} \in B_{r}(\gamma(x))}\left[f\left(T_{1}\right)\right]_{\left\{x^{\prime}\right\}}=\bigcup_{x^{\prime} \in B_{r}(\gamma(x))}\left[f\left(T_{2}\right)\right]_{\left\{x^{\prime}\right\}} \\
& \Rightarrow\left[f\left(T_{1}\right)\right]_{B_{r}(\gamma(x))}=\left[f\left(T_{2}\right)\right]_{B_{r}(\gamma(x))} ;
\end{aligned}
$$

Since $\mathrm{U}_{x^{\prime}} \in_{\mathrm{Br}}(x)\left[f\left(\mathrm{~T}_{1}\right)\right]_{\left\{x^{\prime}\right\}}$ covers $B_{r}(\gamma(x))$.

Lemma 2.5: Being $i-M L D$ has an equivalence relation on tiling spaces.

Proof: Clearly, by definition, i-MLD is reflexive and symmetric. For transitivity, suppose $T_{1}, T_{2} \in \Omega$ and $T_{1}^{\prime}=f\left(T_{1}\right), T_{2}^{\prime}=f\left(T_{2}\right), T_{1}^{\prime \prime}=g\left(T_{2}^{\prime}\right), T_{2}^{\prime \prime}=g\left(T_{2}^{\prime}\right)$,

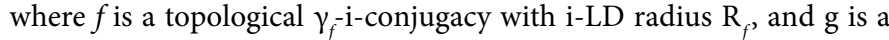
topological $\gamma_{g}$-i-conjugacy with $\mathrm{i}$-LD radius $\mathrm{R}_{\mathrm{g}}$.

If $\left[T_{1}\right]_{B_{R_{f}+R_{g}}(x)}=\left[T_{2}\right]_{B_{R_{f}+R_{g}}(x)}$, then, by using Lemma 2.4 we have:

$\left[T_{1}^{\prime}\right]_{B_{R_{g}}\left(\gamma_{f}(x)\right)}=\left[T_{2}^{\prime}\right]_{B_{R_{g}}\left(\gamma_{f}(x)\right)}$.

Hence, by Definition 2.1, we have:

$\left[T_{1}^{\prime}\right]_{\left\{\gamma_{g}\left(\gamma_{f}(x)\right)\right\}}=\left[T_{2}^{\prime \prime}\right]_{\left\{\gamma_{g}\left(\gamma_{f}(x)\right)\right\}} ;$

and $g \circ f$ is a topological $\left(\gamma_{g} \circ \gamma_{f}\right)$-i-conjugacy.

Remark 2.6: 1 . It is enough to check the $\gamma$-i-LD property of a $\gamma$-ifactor map $f$ at a given point $x$ :

Take an isometry $\phi$ mapping $y$ to $x$, i.e., $\phi(y)=x$, such that $\forall T_{1}, T_{2} \in \Omega$, we have:

$$
\begin{aligned}
{\left[T_{1}\right]_{B_{R}(y)}=\left[T_{2}\right]_{B_{R}(y)} } & {\left[\phi\left(T_{1}\right)\right]_{B_{R}(x)}=\left[\phi\left(T_{2}\right)\right]_{B_{R}(x)} \quad ; \phi\left(T_{1}\right), \phi\left(T_{2}\right) \in \Omega } \\
& {\left[f\left(\phi\left(T_{1}\right)\right)\right]_{\{\gamma(x)\}}=\left[f\left(\phi\left(T_{2}\right)\right)\right]_{\{\gamma(x)\}} } \\
& {\left[\gamma \phi \gamma^{-1} f\left(T_{1}\right)\right]_{\{\gamma(x)\}}=\left[\gamma \phi \gamma^{-1} f\left(T_{2}\right)\right]_{\{\gamma(x) ;} ; } \\
& {\left[\phi \gamma^{-1} f\left(T_{1}\right)\right]_{\{x\}}=\left[\phi \gamma^{-1} f\left(T_{2}\right)\right]_{\{x\}} \text { as } f \text { is } \gamma-\mathrm{i}-\text { factor map } } \\
& {\left.\left[f\left(T_{1}\right)\right]_{\left\{\gamma \phi^{-1}(x)\right\}}=\left[f\left(T_{2}\right)\right]_{\{\gamma \phi}{ }^{-1}(x)\right\} } \\
& {\left[f\left(T_{1}\right)\right]_{\gamma(y)}=\left[f\left(T_{2}\right)\right]_{\gamma(y)} . }
\end{aligned}
$$

2. The properties of $f(T)$ near the point $\gamma(x)$ are determined by the properties of $T$ on a ball around $x$, for all tilings $T$ in the tiling space $\Omega$.

3. Originally, $i-M L D$ was defined using only translations, and for tilings rather than for tiling spaces; (Definition 2.9).

Proposition 2.7: The map $f: \Omega \rightarrow \Omega$ on a tiling space $\Omega$, defined by $f: T \rightarrow \phi(T)$ is a topological conjugacy defining an $i$-MLD homeomorphism; $\phi \in \operatorname{Isom}\left(\mathbb{E}^{\mathrm{n}}\right)$.

Proof: To prove that $f$ is continuous, we use a metric on $\Omega$ constructed from the points $O$ and $\phi(O)$. Since the induced topologies are equal, it would suffice to show that:

$$
\forall \varepsilon>0 \exists \delta>0: d_{O}\left(T, T^{\prime}\right)<\delta d_{\phi(O)}\left(f(T), f\left(T^{\prime}\right)\right)<\varepsilon .
$$

Choose $0<\varepsilon<\ln \left(\frac{3}{2}\right)$ and pick $\delta:=\varepsilon . \mathrm{d}_{0}\left(T, T^{\prime}\right)<\delta$, which means that there exists $\psi, \rho \in \operatorname{Isom}\left(\mathbb{E}^{\mathrm{n}}\right)$, where $d_{O}\left(\psi, i d_{\mathbb{E}^{n}}\right), d_{O}\left(\rho, i d_{\mathbb{E}^{n}}\right)<\frac{1}{2 r}$ with $r:=\frac{1}{e^{\delta}-1}$, such that:

$$
\begin{aligned}
& {[\psi(T)]_{B_{r}(O)}=\left[\rho\left(T^{\prime}\right)\right]_{B_{r}(O)}} \\
& \Rightarrow \phi\left([\psi(T)]_{B_{r}(O)}\right)=\phi\left(\left[\rho\left(T^{\prime}\right)\right]_{B_{r}(O)}\right) \\
& \Rightarrow[\phi \psi(T)]_{B_{r}(\phi(O))}=\left[\phi \rho\left(T^{\prime}\right)\right]_{B_{r}(\phi(O))} \\
& \Rightarrow\left[\phi \psi \phi^{-1} f(T)\right]_{B_{r}(\phi(O))}=\left[\phi \rho \phi^{-1} f\left(T^{\prime}\right)\right]_{B_{r}(\phi(O))} \\
& \Rightarrow d_{\phi(O)}\left(f(T), f\left(T^{\prime}\right)\right)<\ln \left(1+\frac{1}{r}\right)=\delta=\varepsilon
\end{aligned}
$$

since $d_{\phi(O)}\left(\phi \psi \phi^{-1}, i d \mathbb{E}_{n}\right)=d_{0}\left(\psi, i d \mathbb{E}_{n}\right)$ and $d_{\phi(O)}\left(\phi \rho \phi^{-1}, i d \mathbb{E}_{n}\right)=d_{0}\left(\rho, i d \mathbb{E}_{n}\right)$. As required, this shows that $f$ is continuous. Clearly, $f$ is $1-1$, since if we map two different tilings by an isometry $\phi$, we obtain two different tilings; also, $f$ is onto since $f\left(\phi^{-1}(T)\right)=T$. Hence, $f$ is a homeomorphism.

$f$ is a topological $\phi$-i-conjugacy, since

$$
f(\psi(T))=\phi \psi(T)=\phi \psi \phi^{-1}(T)=\phi \psi \phi^{-1} f(\mathrm{~T}) ; \psi \in \operatorname{Isom}\left(\mathbb{E}^{\mathrm{n}}\right)
$$

Finally, we aim to show that $f$ is $\mathrm{i}-\mathrm{MLD}$ for any MLD radius $R$. First, notice that $f$ is $\phi$-i-LD:

$$
\begin{aligned}
{\left[T_{1}\right]_{B_{R}(x)}=\left[T_{2}\right]_{B_{R}(x)} ; \forall T_{1}, T_{2} \in \Omega \quad } & {\left[\phi\left(T_{1}\right)\right]_{B_{R}(\phi(x))}=\left[\phi\left(T_{2}\right)\right]_{B_{R}(\phi(x))} } \\
& {\left[f\left(T_{1}\right)\right]_{\{\phi(x)\}}=\left[f\left(T_{2}\right)\right]_{\{\phi(x)\}} . }
\end{aligned}
$$

Similarly, $f^{1}$ is also $\phi$-i-LD, since $f^{1}$ is given by $\phi^{-1}$. Hence, $f$ is $i$-MLD.

Remark 2.8: There are tiling spaces which are topological i-conjugacies, but not i-MLD. See, for example, the usual Penrose tiling space and the rational Penrose tiling space [1].

\section{MLD Tilings 2.2}

Definition 2.9: If $T$ and $T^{\prime}$ are tilings, $T^{\prime}$ is said to be $\gamma$-i-locally derivable from $T$, if for some finite radius $R$, the properties of $T^{\prime}$ at each point $\gamma \in \mathbb{E}^{\mathrm{n}}$ are determined by the properties of $T$ in a ball of radius $R$ around $x$. More formally, $T^{\prime}$ is $\gamma$-i-locally derivable from $T$ if there exists a radius $R$ such that, for $x \in \mathbb{E}^{\mathrm{n}}$ and $\phi \in \operatorname{Isom}\left(\mathbb{E}^{\mathrm{n}}\right)$, we have the following property:

$$
[T]_{B_{R}(x)}=[\phi(T)]_{B_{R}(x)} \operatorname{implies}\left[T^{\prime}\right]_{\{\gamma(x)\}}=\left[\gamma \phi \gamma^{-1} T^{\prime}\right]_{\{\gamma(x)\}} .
$$

If these implications hold, then the radius $R$ will be called the $i-L D$ radius.

If $T$ is $\gamma^{-1}$-i-locally derivable from $\mathrm{T}^{\prime}$, and $\mathrm{T}^{\prime}$ is $\gamma$-i-locally derivable from $T$, then $T$ and $T^{\prime}$ are called $i-M L D$.

Notice that Remark 2.2 is applied in this section as well.

Notice that S-MLD definition which was mentioned in [2] does not contain conjugation with $\gamma$ (this is necessary for $T$ and $\gamma(T)$ to be MLD); and does not discuss its properties. In particular its connection to MLD of the hulls of $T$ and $\mathrm{T}^{\prime}$, as we will see later on. 
The following example shows that $T$ and $T^{\prime}$ can be translationallyMLD, but not isometrically-MLD.

Example 2.10: The standard lattice tiling $T$, and the slanted lattice tiling $T^{\prime}$ are translationally MLD. $T^{\prime}$ is translationally LD from $T$ because $[T]_{B_{r}(x)}=[T-y]_{B_{r}(x)}$ implies $y \in \mathbb{Z}^{2}$, as the vertices of $T$ are all in $\mathbb{Z}^{2}$. But $y \in \mathbb{Z}^{2}$ also implies, hence we have an equality of patches $\left[T^{\prime}\right]_{\{x\}}=\left[T^{\prime}-y\right]_{\{x\}}$. In the same way we can deduce that $T$ is translationally LD from $\mathrm{T}^{\prime}$. Hence, $T$ and $T^{\prime}$ are translationally-MLD.

On the other hand $T^{\prime}$ is not isometrically LD from $T$ : Rotating $T$ by $90^{\circ}$ around a midpoint $x$ of a tile of $T$ does not change $T$ but, Figure 5 shows that the patches of $T^{\prime}$ and the rotated tiling $T^{\prime}$ covering $x$ are certainly different.

Lemma 2.11: If $T^{\prime}$ is $\gamma$-i-locally derivable from $T$ with $L D$-radius $R$, then for every $r$ :

$$
[T]_{B_{r+R}(x)}=[\phi(T)]_{B_{r+R}(x)}\left[T^{\prime}\right]_{B_{r}(\gamma(x))}=\left[\gamma \phi \gamma^{-1} T^{\prime}\right]_{B_{r}(\gamma(x))} .
$$

Proof: By covering $\mathrm{B}_{\mathrm{r}+\mathrm{R}}(x)$ with balls $\mathrm{B}_{\mathrm{R}}\left(x^{\prime}\right)$, and $\mathrm{B}_{r+R}(y)$ with balls $\mathrm{B}_{\mathrm{R}}\left(y^{\prime}\right)$, where $\left|x^{\prime}-x\right|<r$ and $\left|y^{\prime}-y\right|<r x^{\prime} \in \mathrm{B}_{\mathrm{r}}(x)$ and $y^{\prime} \in \mathrm{B}_{\mathrm{r}}(y)$, we will have:

$$
\begin{aligned}
{[T]_{B_{r+R}(x)}=[\phi(T)]_{B_{r+R}(x)} } & \Rightarrow[T]_{B_{R}\left(x^{\prime}\right)}=[\phi(T)]_{B_{R}\left(x^{\prime}\right)} ; \quad \forall x^{\prime} \in B_{r}(x) \\
& \Rightarrow\left[T^{\prime}\right]_{\left\{\gamma\left(x^{\prime}\right)\right\}}=\left[\gamma \phi \gamma^{-1} T^{\prime}\right]_{\left\{\gamma\left(x^{\prime}\right)\right\}} ; \forall x^{\prime} \in B_{r}(x) \\
& \Rightarrow \bigcup_{x^{\prime} \in B_{r}(\gamma(x))}\left[T^{\prime}\right]_{\left\{\gamma\left(x^{\prime}\right)\right\}}=\bigcup_{x^{\prime} \in B_{r}(\gamma(x))}\left[\gamma \phi \gamma^{-1} T^{\prime}\right]_{\left\{x^{\prime}\right\}} \\
& \Rightarrow\left[T^{\prime}\right]_{B_{r}(\gamma(x))}=\left[\gamma \phi \gamma^{-1} T^{\prime}\right]_{B_{r}(\gamma(x))} .
\end{aligned}
$$

Lemma 2.12: Being $i-M L D$ has an equivalence relation on tilings.

Proof: A tiling is trivially i-MLD with itself. If $T$ and $T^{\prime}$ are i-MLD tilings, then they are each locally derivable from the other; therefore, the property is symmetric. Finally, we show transitivity. Suppose $T, T^{\prime}$ and $T^{\prime \prime}$ are tilings, such that $T^{\prime}$ is $\gamma$-i-locally derivable from $T$ with i-LD radius $R$, and $T^{\prime \prime}$ is $\gamma^{\prime}$-i-locally derivable from $T^{\prime}$ with $i$-LD radius $R^{\prime}$. If $[T]_{B_{R+R^{\prime}}(x)}=[\phi(T)]_{B_{R+R^{\prime}}(x)}$, then, by Lemma 2.11, $\left[T^{\prime}\right]_{B_{R^{\prime}}(\gamma(x))}=\left[\gamma \phi \gamma^{-1} T^{\prime}\right]_{B_{R^{\prime}}(\gamma(x))}$. Hence, by Definition 2.9,

we have:

$\left[T^{\prime \prime}\right]_{\left\{\gamma^{\prime} \gamma(x)\right\}}=\left[\gamma^{\prime} \gamma \phi \gamma^{-1} \gamma^{\prime-1} T^{\prime \prime}\right]_{\left\{\gamma^{\prime \prime}(x)\right\} .}$

Hence, $T^{\prime \prime}$ is $\left(\gamma^{\prime} \gamma\right)$-i-LD from $T$, as required to show transitivity.

Proposition 2.13 : If a tiling $T_{2}$ is obtained by applying an isometry $\theta \in I \operatorname{som}\left(\mathbb{E}^{\mathrm{n}}\right)$ on a tiling $T_{1}$, that is, $T_{2}=\theta\left(T_{1}\right)$; then, $T_{1}$ and $T_{2}$ are $\mathrm{i}-\mathrm{MLD}$.

Proof: Assume that

$$
\begin{aligned}
& {\left[T_{1}\right]_{\mathrm{Br}}(x)=\left[\phi\left(T_{1}\right)\right]_{\mathrm{Br}}(x): } \\
\Rightarrow & {\left[T_{1}\right]_{\{x\}}=\left[\phi\left(T_{1}\right)\right]_{\{x\}} }
\end{aligned}
$$

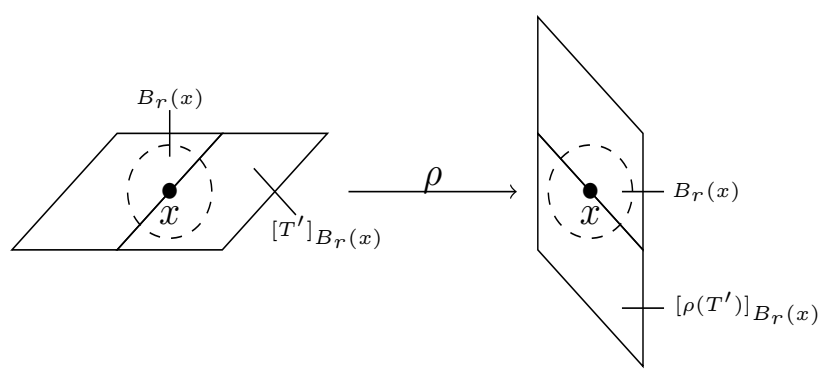

Figure 5: Rotation of tiles counter clockwise by $90^{\circ}$.

$$
\begin{aligned}
& \Rightarrow \theta\left(\left[T_{1}\right]_{\{x\}}\right)=\theta\left(\left[\phi\left(T_{1}\right)\right]_{\{x\}}\right) \\
& \Rightarrow\left[\theta\left(T_{1}\right)\right]_{\{\theta(x)\}}=\left[\theta \phi\left(T_{1}\right)\right]_{\{\theta(x)\}} \\
& \Rightarrow\left[T_{2}\right]_{\{\theta(x)\}}=\left[\theta \phi \theta^{-1} T_{2}\right]_{\{\theta(x)\}} .
\end{aligned}
$$

Hence, $T_{2}$ is $\theta$-i-LD from $T_{1}$. Similarly, $T_{1}$ is $\theta^{-1}$ - $\mathrm{i}-\mathrm{LD}$ from $T_{2}$, and so, $T_{1}$ and $T_{2}$ are i-MLD.

Lemma 2.14 Let $T_{\mathrm{n}} \rightarrow T$ and $S_{\mathrm{n}} \rightarrow S$ convergent series of tilings, such that $\left[T_{n}\right]_{B r(x)}=\left[S_{n}\right]_{B r(x)}$ for some $r>0$. Then,

$$
[T]_{\{x\}}=[S]_{\{x\}} .
$$

Proof: Let $\mathrm{t} \in[T]_{\{x\}}$ be a tile containing $x$. Since $T_{n} \rightarrow T$, we see that there exist tiles $t_{n} \in\left[T_{n}\right]_{B \mathrm{r}\{x\}}$ such that $t_{\mathrm{n}} \rightarrow t$. This implies $t_{n} \in\left[S_{n}\right]_{B \mathrm{r}\{x\}}$ as $\left[T_{n}\right]$ $\operatorname{Br}(x)=\left[\mathrm{S}_{\mathrm{n}}\right]_{\operatorname{Br}\{x\}}$. Next, $t \in[\mathrm{S}]_{\{x\}}$, since $S_{\mathrm{n}} \rightarrow S$. Hence, $[T]_{\{x\}}=[S]_{\{x\}}$.

Lemma 2.15: If $T$ and $T^{\prime}$ are $i-M L D$ tilings, then their associated hulls $\Omega_{T}$ and $\Omega_{T}$ are $i-M L D$.

Proof: Suppose $T^{\prime}$ is $\gamma$-i-LD from $T$ with i-LD-radius $R$, and $T$ is $\gamma^{-1}$-i-LD from $T^{\prime}$ with i-LD-radius $R$. Construct the map $f: \Omega_{\mathrm{T}} \rightarrow \Omega_{\mathrm{T}^{\prime}}$ by setting $f(T)=T^{\prime}$ and extend it to the orbit of $T$ by $f(\phi(T))=\gamma \phi \gamma^{-1}\left(T^{\prime}\right)$.

Claim 1: The map $f: O(T) \rightarrow O\left(T^{\prime}\right)$ is continuous with respect to the topologies induced from the hulls $\Omega_{T}$ and $\Omega_{\mathrm{T}}$.

Proof of claim 1: Let $T_{n} \rightarrow \bar{T}$ be a convergent sequence in $O(T)$. Choose $\phi_{n}, \bar{\phi} \in \operatorname{Isom}\left(\mathbb{E}^{n}\right)$ such that $T_{n}=\phi_{n}(T)$ and $\bar{T}=\bar{\phi}(T)$. Since the orbit $O(T)$ can be a nowhere closed dense subset of the hull $\Omega_{T}$, we cannot assume that $\phi_{n} \rightarrow \bar{\phi}$. Instead we combine the definition of the distance between tilings and that of local derivability.

$T_{n} \rightarrow \bar{T}$ tells us that there exists a large $R \gg 0$ and $\psi_{n}, \bar{\psi}_{n}$ tending to $i d_{\mathbb{E n}}$ such that

$$
\left[\psi_{n} \phi_{n}(T)\right]_{B_{R}(x)}=\left[\bar{\psi}_{n} \bar{\phi}(T)\right]_{B_{R}(x)} .
$$

This implies

$$
[T]_{B_{R}\left(\phi_{n}^{-1} \psi_{n}^{-1}(x)\right)}=\left[\phi_{n}^{-1} \psi_{n}^{-1} \bar{\psi}_{n} \bar{\phi}(T)\right]_{B_{R}\left(\phi_{n}^{-1} \psi_{n}^{-1}(x)\right)} \text {. }
$$

Since $R$ will eventually be much larger than the LD-radius of $T$ and $T^{\prime}$ we may conclude

$$
\left[T^{\prime}\right]_{B_{R^{\prime}}\left(\gamma \phi_{n}^{-1} \psi_{n}^{-1}(x)\right)}=\left[\gamma \phi_{n}^{-1} \psi_{n}^{-1} \bar{\psi}_{n} \bar{\phi} \gamma^{-1}\left(T^{\prime}\right)\right]_{B_{R^{\prime}}\left(\gamma \phi_{n}^{-1} \psi_{n}^{-1}(x)\right)},
$$

for some $R^{\prime}>R / 2$. Hence

$$
\left[\gamma \phi_{n} \gamma^{-1}\left(T^{\prime}\right)\right]_{B_{R^{\prime}}\left(\gamma \psi_{n}^{-1}(x)\right)}=\left[\gamma \psi_{n}^{-1} \bar{\psi}_{n} \bar{\phi}^{-1}\left(T^{\prime}\right)\right]_{B_{R^{\prime}}\left(\gamma \psi_{n}^{-1}(x)\right)} .
$$

Setting $\bar{\psi}_{n}^{\prime}:=\gamma \psi_{n}^{-1} \bar{\psi}_{n}^{-1} \gamma^{-1}$ and possibly further reducing $R^{\prime}$ by an arbitrarily small amount we obtain

$$
\left[\gamma \phi_{n} \gamma^{-1}\left(T^{\prime}\right)\right]_{B_{R^{\prime}}(\gamma(x))}=\left[\bar{\psi}_{n}^{\prime} \gamma \bar{\phi} \gamma^{-1}\left(T^{\prime}\right)\right]_{B_{R^{\prime}}(\gamma(x))}
$$

since $d_{O}\left(\psi_{n}^{-1}, i d_{\mathbb{E}^{n}}\right) \rightarrow 0$. Which implies:

$\gamma \phi_{n} \gamma^{-1}\left(T^{\prime}\right) \rightarrow \gamma \bar{\phi} \gamma^{-1}\left(T^{\prime}\right)$

Claim 2: $f$ is a $\gamma$-i-factor map, that is: $f(\phi(\bar{T}))=\gamma \phi \gamma^{-1} f(\bar{T}) \forall \bar{T} \in \Omega_{T}$.

Proof of claim 2: If $\bar{T}=\lim _{n \rightarrow \infty} \phi_{n}(T)$, then:

$$
\begin{aligned}
f(\bar{T}) & =f\left(\lim _{n \rightarrow \infty} \phi_{n}(T)\right) \\
& =\lim _{n \rightarrow \infty} f\left(\phi_{n}(T)\right) ; \quad \text { byclaim1 } \\
& =\lim _{n \rightarrow \infty} \gamma \phi_{n} \gamma^{-1} f(T) .
\end{aligned}
$$

Now, we have, 


$$
\begin{aligned}
\gamma \phi \gamma^{-1} f(\bar{T}) & =\lim _{n \rightarrow \infty} \gamma \phi \phi_{n} \gamma^{-1} f(T) \\
& =\lim _{n \rightarrow \infty} f\left(\phi \phi_{n} T\right) \\
& =f\left(\phi\left(\lim _{n \rightarrow \infty} \phi_{n}(T)\right)\right) ; \quad \text { byclaim1 } \\
& =f(\phi(\bar{T})) .
\end{aligned}
$$

Claim 3: $f^{-1}$ is a $\gamma^{-1}$-i-factor map.

Proof of claim 3: $f^{-1}$ can be defined by $f^{-1}\left(T^{\prime}\right)=T$ and extended to the orbit, and then to the hull as above. Hence, similar to the proof of claim 2, we will have:

$$
f^{-1}\left(\phi\left(\bar{T}^{\prime}\right)\right)=\gamma^{-1} \phi \gamma f^{-1}\left(\bar{T}^{\prime}\right) \forall \bar{T}^{\prime} \in \Omega_{T^{\prime}}
$$

Claim 4: $f$ is $\gamma$-i-LD.

Proof of claim 4: We first consider the case that $T_{1}, T_{2}$ are in the orbit of $T$, with $T_{1}=\phi(T)$ and $T_{2}=\psi(T)$.

$$
\begin{aligned}
{[\phi(T)]_{B_{R}(x)}=[\psi(T)]_{B_{R}(x)} } & \Rightarrow \phi\left([T]_{B_{R}\left(\phi^{-1}(x)\right)}\right)=[\psi(T)]_{B_{R}(x)} \\
& \Rightarrow[T]_{B_{R}\left(\phi^{-1}(x)\right)}=\left[\phi^{-1} \psi(T)\right]_{B_{R}\left(\phi^{-1}(x)\right)} \\
& \Rightarrow\left[T^{\prime}\right]_{\left\{\gamma\left(\phi^{-1}(x)\right)\right\}}=\left[\gamma \phi^{-1} \psi \gamma^{-1} T^{\prime}\right]_{\left\{\gamma\left(\phi^{-1}(x)\right)\right\}} \\
& \Rightarrow\left[\phi \gamma^{-1} T^{\prime}\right]_{\{x\}}=\left[\psi \gamma^{-1} T^{\prime}\right]_{\{x\}} \\
& \Rightarrow\left[\gamma \phi \gamma^{-1} T^{\prime}\right]_{\{\gamma(x)\}}=\left[\gamma \psi \gamma^{-1} T^{\prime}\right]_{\{\gamma(x)\}} \\
& \Rightarrow[f(\phi(T))]_{\{\gamma(x)\}}=[f(\psi(T))]_{\{\gamma(x)\}} .
\end{aligned}
$$

Now, we can prove the general case:

Assume that $T_{1}=\lim _{\mathrm{n} \rightarrow \infty} \phi_{n}(T), T_{2}=\lim _{\mathrm{n} \rightarrow \infty} \psi_{n}(T)$, and $\left[T_{1}\right]_{B_{R}(x)}=\left[T_{2}\right]_{B_{R}(x)}$ Furthermore, define the distance on $\Omega_{\mathrm{T}}$ using the origin $x$, and the distance on $\Omega_{\mathrm{T}^{\prime}}$ using the origin $\gamma(x)$. Then, for a given $R^{\prime}>R$, $d\left(T_{1}, \phi_{\mathrm{n}}(T)\right) \rightarrow 0$ implies that for $n \gg 0$, there exists $\phi_{n}^{\prime}, \phi_{n}^{\prime \prime} \in \operatorname{Isom}\left(\mathbb{E}^{n}\right)$, such that $d_{x}\left(\phi_{n}^{\prime}, i d_{\mathbb{E}^{n}}\right), d_{x}\left(\phi_{n}^{\prime \prime}, i d_{\mathbb{E}^{n}}\right)$ is arbitrarily small and

$$
\left[\phi_{n}^{\prime} T_{1}\right]_{B_{R^{\prime}}(x)}=\left[\phi_{n}^{\prime \prime} \phi_{n} T\right]_{B_{R^{\prime}}(x)} \text {. }
$$

Similarly, for $n \gg 0$, there exists $\psi_{n}^{\prime}, \psi_{n}^{\prime \prime} \in \operatorname{Isom}\left(\mathbb{E}^{n}\right)$, such that $d_{x}\left(\psi_{n}^{\prime}, i d_{\mathbb{E}^{n}}\right)$ and $d_{x}\left(\psi_{n}^{\prime \prime}, i d_{\mathbb{E}^{n}}\right)$ are arbitrarily small and

$$
\left[\psi_{n}^{\prime} T_{2}\right]_{B_{R^{\prime}}(x)}=\left[\psi_{n}^{\prime \prime} \psi_{n} T\right]_{B_{R^{\prime}}(x)} .
$$

These two equalities imply that:

$$
\left[T_{1}\right]_{B_{R^{\prime}}\left(\phi_{n}^{\prime-1}(x)\right)}=\left[\phi_{n}^{\prime-1} \phi_{n}^{\prime \prime} \phi_{n} T\right]_{B_{R^{\prime}}\left(\phi_{n}^{\prime-1}(x)\right)}
$$

and

$$
\left[T_{2}\right]_{B_{R^{\prime}}\left(\psi_{n}^{\prime-1}(x)\right)}=\left[\psi_{n}^{\prime-1} \psi_{n}^{\prime \prime} \psi_{n} T\right]_{B_{R^{\prime}}\left(\psi_{n}^{\prime-1}(x)\right)} .
$$

Since $R^{\prime}>R$ and $\phi_{n}^{\prime}, \psi_{n}^{\prime}$ are arbitrarily close to $i d_{\mathbb{E n}}$, we have $B_{R}(x) \subset B_{R^{\prime}}\left(\phi_{n}^{\prime-1}(x)\right)$ respectively $B_{R^{\prime}}\left(\psi_{n}^{\prime-1}(x)\right)$; hence,

$$
\begin{aligned}
& {\left[T_{1}\right]_{B_{R}(x)}=\left[\phi_{n}^{\prime-1} \phi_{n}^{\prime \prime} \phi_{n} T\right]_{B_{R}(x)}} \\
& \text { and } \\
& {\left[T_{2}\right]_{B_{R}(x)}=\left[\psi_{n}^{\prime-1} \psi_{n}^{\prime \prime} \psi_{n} T\right]_{B_{R}(x)} .}
\end{aligned}
$$

Therefore, by assumption, $\bar{\phi}_{n}:=\phi_{n}^{\prime-1} \phi_{n}^{\prime \prime} \phi_{n}$ and $\bar{\psi}_{n}:=\psi_{n}^{\prime-1} \psi_{n}^{\prime \prime} \psi_{n}$ are close to $\phi_{n}$ and $\psi_{n}$ such that:

$$
\left[\bar{\phi}_{n} T\right]_{B_{R}(x)}=\left[\bar{\psi}_{n} T\right]_{B_{R}(x)} \text {. }
$$

Now, using the special case in the calculation in eqn.(8) in the beginning of the proof of claim 4 , we conclude:

$$
\left.\left[f\left(\bar{\phi}_{n}(T)\right)\right)\right]_{\{\gamma(x)\}}=\left[f\left(\bar{\psi}_{n}(T)\right)\right]_{\{\gamma(x)\}} .
$$

Notice that $\lim _{n \rightarrow \infty} \bar{\phi}_{n} T=T_{1}$ and $\lim _{n \rightarrow \infty} \bar{\psi}_{n} T=T_{2}$, hence, according to Lemma 2.14 we have,

$$
\left[f\left(\mathrm{~T}_{1}\right)\right]_{\gamma(x)}=\left[f\left(\mathrm{~T}_{2}\right)\right]_{\gamma(x)}
$$

as claimed.

In a completely analogous way, we can show that the inverse $f^{1}$ is $\gamma^{-1}-i$-LD.

Lemma 2.16: If $\Omega$ and $\Omega^{\prime}$ are $i-M L D$ tiling spaces with $i-M L D$ homeomorphism $f: \Omega \rightarrow \Omega$, then $T$ and $f(T)$ are $i-M L D$, for all tilings $T \in \Omega$.

Proof: Suppose $\Omega_{\mathrm{T}}$ and $\Omega_{\mathrm{T}^{\prime}}$ are tiling spaces and $\gamma$-i-LD with the map $f: \Omega \rightarrow \Omega^{\prime}$. If $T \in \Omega$, the properties of $f(T)$ near the point $\gamma(x)$ are determined by the properties of $T$ on some balls around $x$. Hence, the tilings $T$ and $f(T)$ are i-MLD. In more detail,

$$
\begin{aligned}
{[T]_{B_{R}(x)}=[\phi(T)]_{B_{R}(x)} } & \Rightarrow[f(T)]_{\{\gamma(x)\}}=[f(\phi(T))]_{\{\gamma(x)\}} \text { by Defnition } 2.1 \\
& \Rightarrow[f(T)]_{\{\gamma(x)\}}=\left[\gamma \phi \gamma^{-1} f(T)\right]_{\{\gamma(x)\}} .
\end{aligned}
$$

Therefore, $T$ and $f(T)$ are i-MLD, for all tilings $T \in \Omega$.

\section{References}

1. Sadun L (1997) Topology of tiling spaces. University Lecture Series. University of Toronto Press, 46.

2. Schlottmannt M, Baaket M, Jamis PD (1991) Quasiperiodic tilings with tenfold symmetry and equivalence with respect to local derivability. J Phys A: Math Gen 24: 4637-4654.

3. Burris SN (1981) H.P. Function spaces. Springer, millinume edition.

4. Farkas DR (1981) Crystallographic groups and their mathematics. Rocky Mountain Mathematics Consortium 11: 511-551.

5. Sadun L (2006) Tilings, tiling spaces and topology. Philos Mag 86: 875-881.

6. Radin C (1994) The pinwheel tilings of the plane. Ann of Math 139: 661-702.

7. Grunbaum B, Shephard GC (1989) Tilings and patterns. W. H. Freeman and Company, New York.

8. Grunbaum B (2003) Convex polytopes. Springer-Verlag New York 221: 471

9. Axler $S$ (1997) Linear algebra done right. Undergraduate Texts in Mathematics. Springer-Verlag, New York.

10. Baake M, Grimm U (2013) Aperiodic order. A mathematical invitation. Cambridge University Press

11. Micheal SO (2007) Metric spaces. Springer-Verlag London: 304.

12. Senechal M (1996) Quasicrystals and geometry. University of Cambridge: 286 